\title{
METACERCÁRIAS DEAUSTRODIPLOSTOMUM COMPACTUM (LUTZ, 1928) EM PEIXES DE LAGOS DE VÁRZEA DA AMAZÔNIA BRASILEIRA
}

\author{
José Francalino VITAL ${ }^{1}$, Germán Augusto Murrieta MOREY ${ }^{1}$, Natália Brandão PEREIRA , José Celso de \\ Oliveira MALTA ${ }^{1}$ \\ 1 Instituto Nacional de Pesquisas da Amazônia, Laboratório de Parasitologia de Peixes Av. André Araújo 2936, Petrópolis, \\ CEP-69.067-375, Manaus, Amazonas, Brasil. Email: ffvital@yahoo.com.br
}

\section{RESUMO}

O presente estudo investiga a presença de diplostomídeos parasitas em dois peixes curimatídeos, Potamorhina latior e Potamorhina pristigaster, além de listar todos os peixes reportados como hospedeiros para Austrodiplostomum compactum na Amazônia brasileira. Os peixes foram coletados em oito lagos de várzea do rio Solimões, no período de fevereiro de 2013 a dezembro de 2015. Foram analisados cem indivíduos de $P$. latior e sessenta e nove indivíduos de $P$. pristigaster, nos quais foram encontrados nos olhos dois espécimes de $A$. compactum em $P$. latior e apenas um espécime em $P$. pristigaster. Com os novos registros um total de oito espécies de peixes são citadas como hospedeiras para $A$. compactum na Amazônia brasileira incluindo Acestrorhynchus falcirostris, Cichla monoculus, Plagioscion squamosissimus Pterygoplichthys pardalis e Pygocentrus nattereri. As infecções ocorrem em diferentes órgãos: brânquias, musculatura, mesentério, olhos, gônadas, cavidade visceral e rins. Verifica-se que A. compactum apresenta baixa especificidade pelos hospedeiros e estima-se que os baixos índices parasitários associados a amostras de pequeno tamanho subestimem as diversas outras espécies de peixes amazônicos que albergam estes diplostomídeos.

PALAVRAS CHAVE: Austrodiplostomum; Diplostomidae; Lagos de várzea; Parasitos de peixes.

\section{AUSTRODIPLOSTOMUM COMPACTUM (LUTZ, 1928) METACERCARIAE IN FISH FROM BRAZILIAN AMAZON FLOODPLAIN LAKES}

\begin{abstract}
This study investigates the presence of diplostomids parasites in two curimatids fish, Potamorhina latior and Potamorhina pristigaster and lists all the fish reported as hosts for Austrodiplostomum compactum in the Brazilian Amazon. Fish were collected in 8 Solimões river floodplain lakes, from February 2013 to December 2015. One hundred individuals of $P$. latior and sixty-nine individuals of $P$. pristigaster were analyzed, in which were found two specimens of $A$. compactum in the eyes of $P$. latior and only one specimen in the eye of P. pristigaster. With these two new records, a total of eight fish species are cited as hosts species for $A$. compactum in the Brazilian Amazon including: Acestrorhynchus falcirostris, Cichla monoculus, Plagioscion squamosissimus Pterygoplichthys pardalis and Pygocentrus nattereri. The Infections occur in different organs: gills, muscles, mesentery, eyes, gonads, visceral cavity and kidneys. A. compactum has low specificity for fish host species and it is estimated that low parasitic indices associated with small samples underestimate other Amazonian fish species that harbor these parasites.
\end{abstract}

KEYWORDS: Austrodiplostomum; Diplostomidae; Fish parasites; Floodplain lakes. 


\section{INTRODUÇÃO}

Metacercárias de diplostomídeos são amplamente distribuídas na região Neotropical, elas têm um ciclo de vida que depende de três hospedeiros para ser completado: um molusco (hospedeiro intermediário); um peixe (segundo hospedeiro intermediário) ou, raramente um anfíbio (hospedeiro paratênico) e um peixe piscívoro como hospedeiro definitivo. Parasitas adultos são encontrados no intestino das aves piscívoras, seus ovos são depositados nas fezes das aves e posteriormente na água a larva miracídio eclode e nada ativamente a procura de um molusco, onde penetra e se desenvolve em esporocisto mãe, reproduzindo-se assexuadamente para produzir esporocistos filhas. Cada esporocisto filha se reproduz assexuadamente para produzir cercárias, que são a fase infectante (Karvonen et al., 2006; Violante-Gonzalez et al., 2009). Os moluscos infectados liberam as cercarias que infectam os peixes ativamente, geralmente através da boca ou brânquias, embora a penetração também possa ocorrer pelas nadadeiras ou superfície do corpo (Grobbelaar et al., 2014). Aves piscívoras adquirem as metacercárias ao consumir peixes infetados (Chappell et al. 1994;).

As metacercárias de Diplostomidae podem alterar o comportamento dos peixes (Seppala et al., 2005) e provocar doenças, como patologias oculares severas associadas a cataratas, cegueira e em alguns casos à morte (Valtonen \& Gibson, 1997). A formação de cataratas em peixes infectados por metacercárias de Diplostomidae é mais intensa depois que estas completam seu desenvolvimento, incrementando assim a susceptibilidade dos peixes à predação, garantindo desta forma a transmissão das metacercárias para o hospedeiro definitivo (Mouritsen \& Poulin 2003).

Metacercárias do diplostomídeo Austrodiplostomum spp. infectam diversas espécies de peixes da América do Sul (Zica et al., 2011). No Brasil Austrodiplostomum compactum (Lutz, 1928) tem sido reportado em 36 espécies de peixes de água doce, em diversos sítios de infecção, como brânquias, olhos, bexiga natatória, cérebro e musculatura (Ramos et al., 2013), porém estes dados incluem apenas 2 hospedeiros da Bacia Amazônica, região com a maior diversidade de peixes do mundo. Assim este estudo reporta a ocorrência de metacercárias de A. compactum em Potamohina latior (Spix \& Agassiz, 1829) e Potamorhina pristigaster (Steindachner, 1876), além de listar todas as outras espécies reportadas como hospedeiras de $A$. compactum em peixes de lagos de várzea amazônicos.

\section{MATERIALE MÉTODOS}

Os peixes foram capturados no período de fevereiro de 2013 a dezembro de 2015, em oito lagos de várzea, cinco do rio Solimões: Catalão ( $3^{\circ} 09^{\prime} 47^{\prime}$ "

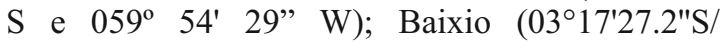
$\left.60^{\circ} 04^{\prime} 29.6^{\prime \prime} \mathrm{O}\right)$ no município de Iranduba; Preto $\left(03^{\circ} 21^{\prime} 17.1^{\prime \prime S} / 60^{\circ} 37^{\prime} 28.6^{\prime \prime O}\right)$ em Iranduba; Ananá (035' $\left.54.8^{\prime \prime} \mathrm{S} / 6^{\circ} 40^{\prime} 18.4^{\prime \prime O}\right)$ em Anori; Araçá ( $\mathrm{S} 03^{\circ} 45^{\prime}$ 04.3" $\left.\mathrm{S} / 6^{\circ} 21^{\prime} 25.9^{\prime \prime} \mathrm{O}\right)$ em Codajás; Maracá (0350'32.8"S/ 62³4'32.4"O) em Coari e um lago do rio Purus, São Tomé (0349'39.0"S/ $61^{\circ} 25^{\prime} 24.6^{\prime \prime} \mathrm{O}$ ). Os peixes foram capturados com redes de espera e ainda no campo foram identificados, pesados e medidos. As brânquias e cabeça foram removidas e preservadas em frascos de vidro com formalina a 5\% e o trato digestório fixado em álcool $70 \%$ aquecido. No laboratório os espécimes encontrados foram corados em carmalumen de Mayer, desidratados em série alcoolica, clarificados em salicilato de metila e montados em lâminas permanentes com bálsamo do Canadá (Amato et al., 1991). Os índices parasitários foram calculados e analisados de acordo com Bush et al. (1997). A identificação taxonômica foi feita de acordo com Travassos et al. (1969) e Kohn et al. (1995). Em adição dados de estudos com diplostomídeos realizados na Bacia Amazônica com foram revisados para compor a listagem de hospedeiros para A. compactum.

\section{RESULTADOS}

Foram analisados 100 indivíduos de P. latior e 69 indivíduos de $P$. pristigaster. Destes apenas 2 espécimes de $P$. latior e 1 de $P$. pristigaster estavam infectados com metacercária de A. compactum.

Austrodiplostomum compactum (Figura 1). Descrição baseada em três espécimes: Corpo foliáceo oval, ligeiramente côncavo na face ventral, 1,876 (1,644-2.148) comprimento, 743 (594- 796) largura máxima. Segmento cônico reduzido na região posterior,186 (154-198) comprimento, 237 (199-247) largura. Ventosa oral subterminal, 78 (66-83) comprimento e 67 (59-75) largura; duas pseudoventosas laterais na região anterior do corpo; ventosa ventral ausente. Faringe muscular oval, 88 (78-93) comprimento, 69 (61-75) largura; esôfago curto; cecos intestinais terminando próximo da extremidade da porção anterior do corpo. Órgão tribocítico oval localizado no final do terço posterior da porção anterior do corpo, 353 (291-372) comprimento e 147 (134-158) largura. Primórdios genitais presentes, na forma de duas massas celulares localizadas na extremidade da região posterior do corpo, após o órgão tribocítico. 
A

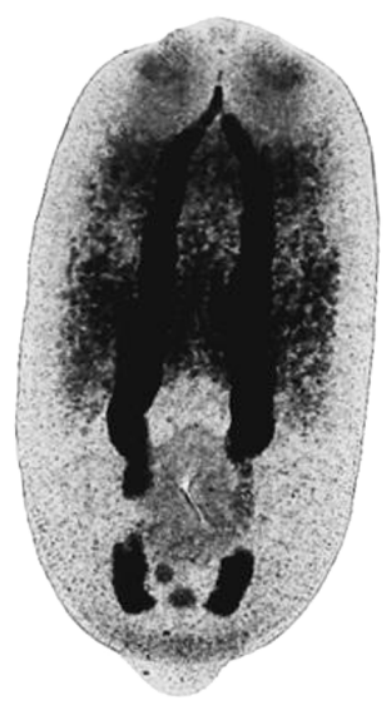

B

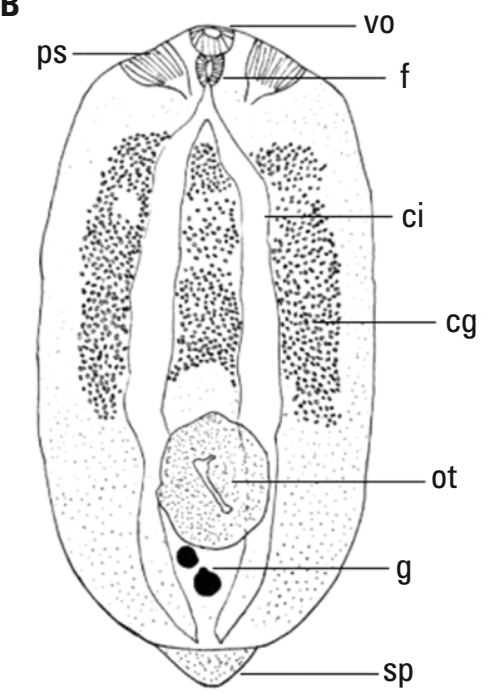

Figura 1. Metacercária de $A$. compactum parasita de Potamorhina spp. coletadas em lagos de várzea no Amazonas, Brasil. A. Fotografía do corpo. B. Esquema mostrando os diferentes órgãos. $\mathrm{vo}=$ ventosa oral; $\mathrm{ps}=$ pseudoventosa; $\mathrm{f}=$ faringe; $\mathrm{ci}=$ cecos intestinais; $\mathrm{cg}=$ células glandulares; ot = órgão tribocítico; $\mathrm{g}=$ gônadas; $\mathrm{sp}=$ segmento posterior do corpo. Escala da barra $500 \mu \mathrm{m}$.

$\mathrm{Na}$ Amazônoa brasileira são registrados oito peixes parasitados por A. compactum: P. latior, $P$. pristigaster, Pygocentrus nattererii Kner, 1858; Pterygoplichthys pardalis (Castelnau, 1855); Acestrorhynchus falcirostris (Cuvier, 1819); Plagioscion squamosissimus (Heckel, 1840); Cichla monoculus Agassiz, 1831 e Hoplias malabaricus (Bloch, 1794). Os índices parasitários são apresentados na tabela 1 e os dados morfométricos das metacercárias são apresentados na tabela 2 .

\section{DISCUSSÃO}

$\mathrm{Na}$ amazônia até o presente foram registrados 6 hospedeiros para A. compactum, com diferentes níveis de infestação. Estes registros mostram diferentes locais de infecção pelo parasita no corpo do hospedeiro. Apesar de uma aparente preferência para os olhos do peixe (incluindo retina humor vítreo, humor aquoso e cristalino) podem ser encontradas parasitando musculatura, estômago, mesentério, rins, gônadas e brânquias. Tendo em consideração a presença de $A$. compactum em diferentes espécies de peixes de várias famílias, assume-se que este parasita apresenta baixa especificidade pelo hospedeiro. No presente trabalho, este parasita é registrado pela primeira vez parasitando $P$. latior e $P$. pristigaster, aumentando assim, o número de hospedeiros conhecidos para esta espécie.
Na Amazônia os maiores níveis de infecção são registrados para $P$. squamosissimus, indicando uma forte susceptibilidade por este parasita e refletindo a presença e abundância de outros organismos que atuam como hospedeiros intermediários $\mathrm{e}$ definitivos de $A$. compactum. No presente estudo, os baixos níveis de infecção podem indicar uma maior resistência por Potamorhina spp. ou talvez as estratégias de vida utilizadas por estas espécies não são as melhores para que $A$. compactum garanta o sucesso no seu ciclo de vida.

A comparação dos dados morfométricos de $A$. compactum de diferentes hospedeiros revela dados discrepantes. Muitos fatores podem atuar para produzir variações significativas na morfometria das metacercárias, tais como, a espécie hospedeira, o tamanho e a idade do hospedeiro, o tamanho da população associada ao crescimento intesidadedependente, condições da metacercária quando fixada e a tecnica utilizada na fixação (Brown et al., 2003).

Apesar de A. compactum não ter sido encontrado em muitos hospedeiros com a fauna parasitária já estudada na Bacia Amazônica, muitos hospedeiros podem ainda ser registrados, pois os baixos indíces parasitários em muitos hospedeiros, associados a amostras de pequeno tamanho podem ter subestimado a presença deste parasita. 


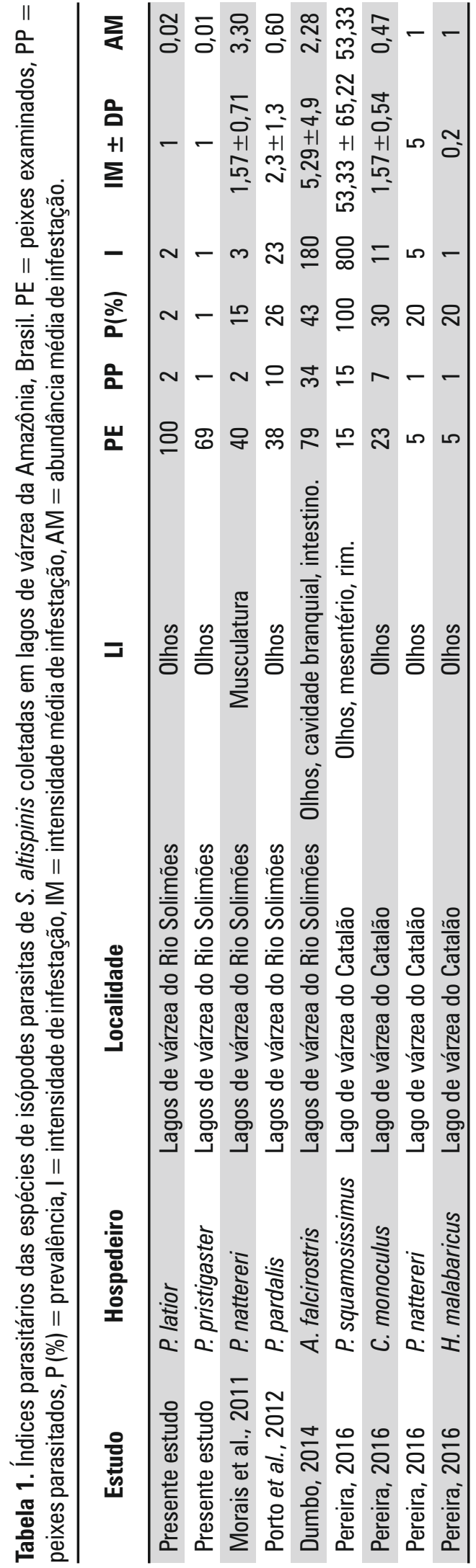

\section{REFERÊNCIAS BIBLIOGRÁFICAS}

Amato, J.F.R. 1991. Protocolos para laboratório coleta e processamento de parasitas do pescado. Imprensa Universitária, Universidade Federal do Rio de Janeiro, Rio de Janeiro, Brasil. 81 p.

Brown, S.P.; De Lorgeril, J.; Joly C.; Thomas, F. 2003. Field evidence for densitydependent effects in the trematode Microphallus papillorobustus in its manipulated host, Gammarus insensibilis. Journal of Parasitology, 89(4): 668- 672.

Bush, A.O.; Lafferty, K.D.; Lotz, J.M.; Shostak, A.W. 1997. Parasitology meets ecology on its own terms: Margolis et al. Revisited. Journal of Parasitology, 83(4): 575 - 583.

Chabros, M.; Pojmanska, T. 1993. Parasites of common carp and three introduced cyprinid fish in pond culture. Acta parasitologica, 38(3), 101108.

Chappell, L.H.; Hardie, L.J.; Secombes, C.J. 1994. Diplostomiasis: the disease and host-parasite interactions. Parasitic diseases of fish, 5: 59-86.

Dumbo, J.C. 2014. Espécies de metazoários parasitos de Acestrorhynchus falcirostris (Cuvier, 1819) (Characiformes: Acestrorhynchidae) de lagos de várzea da Amazônia. Dissertação de Mestrado. INPA, Manaus, 201p.

Grobbelaar, A.; Van As, L.L.; Butler, H.J.; Van As, J.G. 2014. Ecology of diplostomid (Trematoda: Digenea) infection in freshwater fish in southern Africa. African Zoology, 49(2): 222-232.

Karvonen, A.; Terho, P.; Seppälä, O.; Jokela, J.; Valtonen, E.T. 2006. Ecological divergence of closely related Diplostomum (Trematoda) parasites. Parasitology, 133(02): 229-235.

Kohn, A.; Fernandes, B. M.; Baptista-Farias, M.D.F.D. 1995. Metacercariae of Diplostomum (Austrodiplostomum) compactum (Trematoda, Diplostomidae) in the eyes of Plagioscion squamosissimus (Teleostei, Sciaenidae) from the reservoir of the Hydroeletric Power Station of Itaipu, Brazil. Memórias do Instituto Oswaldo Cruz, 90(3): 341-344.

Lacerda, A.C.F.; Takemoto, R.M.; Tavares-Dias, M.; Poulin, R.; Pavanelli, G.C. 2012. Comparative parasitism of the fish Plagioscion squamosissimus in native and invaded river basins. J Parasitol 2012; 98(4): 713-717.

Machado, P.M.; Takemoto, R.M.; Pavanelli, G.C. 2005. Diplostomum (Austrodiplostomum) compactum (Lutz, 1928) (Platyhelminthes, Digenea) metacercariae in fish from the 


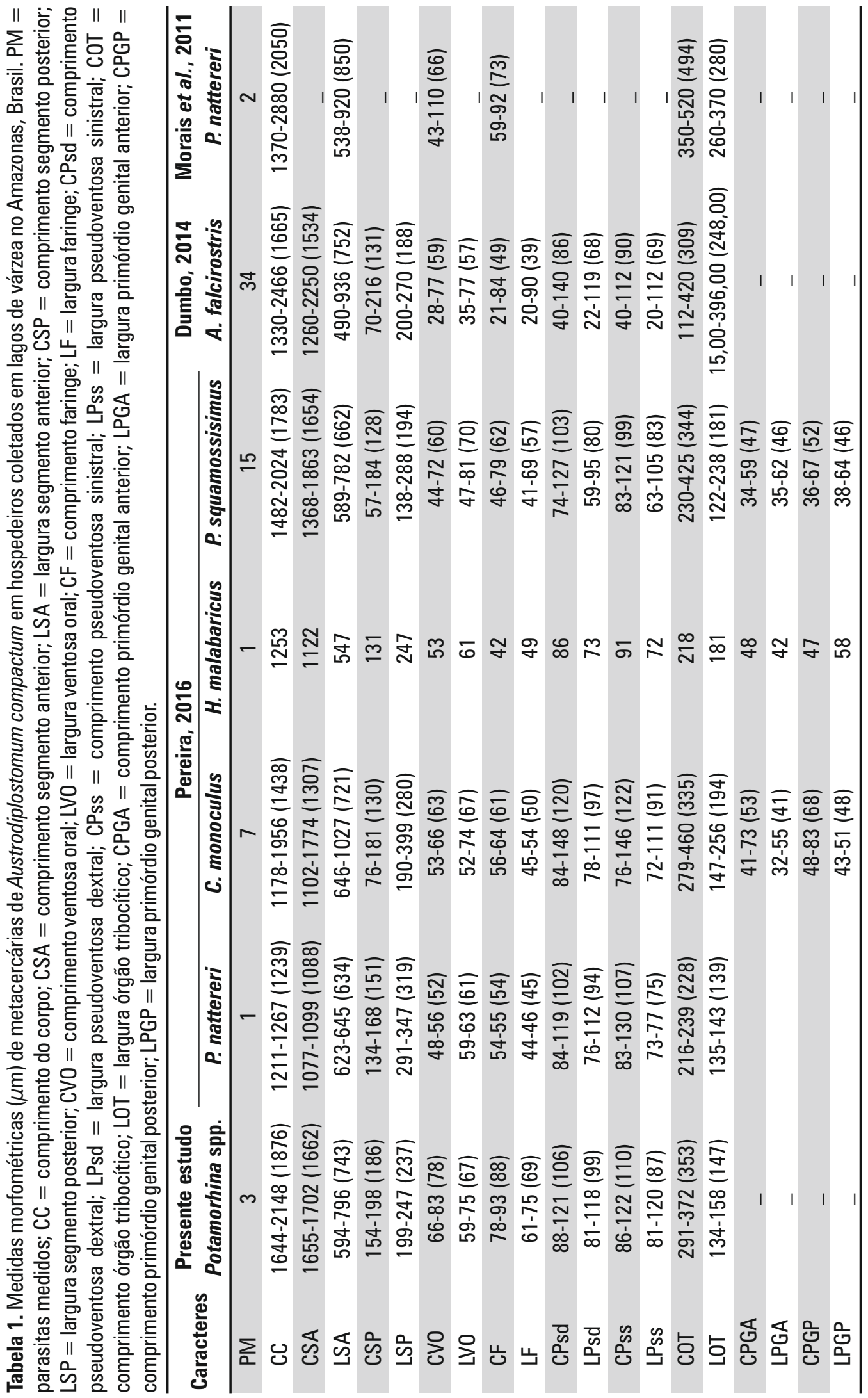


floodplain of the Upper Paraná River, Brazil. Parasitology Research, 97(6): 436-444.

McFarland, L.H.; Mouritsen, K.N.; Poulin, R. 2003. From first to second and back to first intermediate host: the unusual transmission route of Curtuteria australis (Digenea: Echinostomatidae). Journal of Parasitology, 89(3): 625-628.

Morais, A.M. 2011. Biodiversidade de parasitos da piranha vermelha Pygocentrus nattereri (Kner, 1858) (Characiformes; Serrasalmidae) e sua avaliação como bioindicadores na Amazônia Central. Tese de doutorado. INPA, Manaus, 243p.

Pereira, N.R.B. 2016. As espécies parasitas com potencial zoonótico em peixes amazônicos. Dissertação de mestrado. UFAM, Manaus, 152p.

Porto, D.B.; Vital, J.F.; Souza, A.K.; Morais, A. M.; Varella, A.M.B.; Malta J.C. 2012. Metazoários parasitos de Pterygoplichthys pardalis (Castelnau, 1855) (Siluriformes: Loricariidae) da Amazônia central, Brasil. Revista Brasileira de Zoociências, 14(1): 35-40.

Ramos, I.P.; Franceschini, L.; Zago, A.C.; Zica, É.D.O.P.; Wunderlich, A.C.; Carvalho, E.D.; Silva, R.J.D. 2013. New host records and a checklist of fishes infected with Austrodiplostomum compactum (Digenea: Diplostomidae) in Brazil. Revista Brasileira de Parasitologia Veterinária, 22(4): 511-518.
Seppälä, O.; Karvonen, A.; Valtonen, E.T. 2005. Manipulation of fish host by eye flukes in relation to cataract formation and parasite infectivity. Animal Behaviour, 70(4): 889-894.

Travassos, L.; Freitas, J.T.D.; Kohn, A. 1969. Trematódeos do Brazil. Memórias do Instituto Oswaldo Cruz, 67(1): 1-886.

Valtonen, E.T.; Gibson, D.I. 1997. Aspects of the biology of diplostomid metacercarial (Digenea) populations occurring in fishes in different localities of northern Finland. In Annales Zoologici Fennici (pp. 47-59). Finnish Zoological and Botanical Publishing Board.

Violante-González, J.; García-Varela, M.; RojasHerrera, A.; Guerrero, S.G. 2009. Diplostomiasis in cultured and wild tilapia Oreochromis niloticus in Guerrero State, Mexico. Parasitology research, 105(3): 803-807.

Zica, E.O.P.; Brandão, H.; Zawadzki, C.H.; Nobile, A.B.; Carvalho, E.D.; Da Silva, R.J. 2011. The occurrence of Austrodiplostomum compactum (Lutz, 1928) (Digenea: Diplostomidae) metacercariae in the eyes of loricariid fish (Siluriformes: Osteichthyes: Loricariidae) from Brazil. Journal of Helminthology, 85(01): 73-79.

Recibido: 1 de Agosto del 2016

Aceptado para publicación: 30 de Agosto del 2016 